\title{
Abordagem comportamental como estratégia para 0 tratamento da obesidade na Atenção Primária à Saúde
}

Daniel Demétrio Faustino-Silva, Natália Miranda Jung, Luiza Laubert La Porta

\section{RESUMO}

Motivação: 0 aumento da prevalência de excesso de peso e obesidade é uma realidade mundial. A busca por novas estratégias de tratamento se torna importante, uma vez que abordagens convencionais talvez não estejam sendo efetivas. A abordagem comportamental em nutrição pode ser uma alternativa como nova forma de cuidado já que possibilita também uma maior compreensão do comportamento relacionado ao ato de comer excessivo. Dessa maneira, o objetivo do trabalho foi a elaboração de um material científico que promova conhecimento sobre a abordagem comportamental aplicada em nutrição voltada para pessoas com obesidade.

Método: Revisão bibliográfica, resumo de conteúdo, adaptação e criação de material didático.

Resultados: A partir da revisão teórica realizada foi desenvolvida cartilha, com elementos teóricos e práticos, que visa a oferecer um material de cunho teórico e prático sobre abordagem comportamental e a aplicação de suas técnicas em pacientes obesos no âmbito de ações coletivas de saúde na APS.

Conclusões: A elaboração de um material científico promove conhecimento aplicado em nutrição voltada para pessoas com obesidade.

Palavras-chave: Atenção Primária à Saúde; Comportamento Alimentar; Obesidade; Educação Alimentar e Nutricional; Terapia Cognitivo-comportamental.
Revista da Rede APS 2019

Publicada em: 17/12/2019

DOI:10.14295/aps.v1i3.43

Daniel Demétrio FaustinoSilva

(Grupo Hospitalar Conceição, Porto Alegre, Rio Grande do Sul, Brasil);

Natália Miranda Jung (Grupo Hospitalar Conceição, Porto Alegre, Rio Grande do Sul, Brasil);

Luiza Laubert La Porta (Grupo Hospitalar Conceição, Porto Alegre, Rio Grande do Sul, Brasil);

Correspondência para: Daniel Demétrio Faustino-Silva ddemetrio@gmail.com 


\section{INTRODUÇÃO}

A população brasileira passou por importante transição nutricional nas últimas quatro décadas, com queda da desnutrição e aumento do excesso de peso, em todas as idades e classes de renda (ABESO, 2016). Sendo a obesidade um agravo multifatorial, (WORLD HEALTH ORGANIZATION, 2000), cuja etiologia é resultado da complexa interação entre genes, ambiente, estilo de vida e fatores emocionais (ABESO, 2016), torna-se importante optar por abordagens a longo prazo e com estratégias múltiplas (BUTLAND et al., 2007).

A abordagem convencional em atendimentos de nutrição rotula e simplifica o contexto do indivíduo, focalizando em prescrições baseadas em metas e planos de ação em curto prazo. Ainda, com enfoque em alimentos e nutrientes, propõe um relacionamento mínimo entre sujeito e nutricionista. Por outro lado, a abordagem comportamental reconhece o contexto, valida aspectos emocionais, fisiológicos e sociais relacionados ao ato da alimentação, propondo-se a compreender crenças e sentimentos que o indivíduo identifica em relação aos alimentos. Assim, entende-se que o tratamento dietético se torna mais bem-sucedido quando aliado a um propósito de modificação comportamental (ABESO, 2016). Para essa abordagem existem ferramentas e estratégias de intervenção que favorecem a promoção de mudanças, tais como a Entrevista Motivacional (EM), o comer intuitivo e o comer com atenção plena. Dessa forma, a promoção de saúde é estabelecida retomando a relação positiva entre alimentação e indivíduo, respeitando suas subjetividades, com intervenções em longo prazo reconhecidas por ambos atores (ALVARENGA et al., 2015).

Ações coletivas de educação em saúde aumentam a autonomia, fortalecem a relação entre os sujeitos assim como possibilitam maior empoderamento e conhecimento. A Atenção Primária à Saúde (APS), também denominada de Atenção Básica no Brasil, enquanto porta de entrada do SUS e serviço mais próximo da população, conta com abordagens de educação em saúde de âmbito coletivo (BRASIL.
MINISTÉRIO DA SAÚDE., 2006). Com objetivo de atuação multiprofissional, intersetorial e transdisciplinar, ações de Educação Alimentar e Nutricional (EAN) também se propõem a desenvolver a autonomia e voluntariedade ante os hábitos alimentares (BRASIL. MINISTÉRIO DO DESENVOLVIMENTO SOCIAL E COMBATE À FOME, 2012).

Dessa maneira, o presente trabalho teve por objetivo a elaboração de uma cartilha que visa a oferecer um material de cunho teórico e prático sobre abordagem comportamental e a aplicação de suas técnicas em pacientes obesos no âmbito de ações coletivas de saúde na APS, assim como propiciar uma reflexão mais aprofundada sobre o papel do profissional de saúde no enfrentamento da obesidade.

\section{MÉTOdos}

Inicialmente foi realizada uma revisão da literatura nas bases de dados eletrônicas PubMed, SciELO, MEDLINE e LILACS, assim como em livros especializados no assunto. Foram utilizados termos de busca relacionados à educação alimentar e nutricional e comportamento alimentar. Foram selecionados os trabalhos mais abrangentes em língua portuguesa, espanhola e inglesa, por conveniência.

Com os dados obtidos na revisão, pode-se elaborar a cartilha com embasamento científico, sendo o primeiro capítulo de cunho teórico e o segundo, prático. A parte prática é composta por dinâmicas, algumas foram criadas pelos autores assim como outras adaptadas de e referenciadas.

Dessa maneira elaborou-se a cartilha "DA FOME AO COMPORTAMENTO: Abordagem comportamental aplicada em Grupos de Nutrição na Atenção Básica" sendo inicialmente proposta como trabalho de conclusão de residência multiprofissional em saúde do Grupo Hospitalar Conceição-GHC (Porto Alegre, RS), a qual foi reduzida a fim de possibilitar a publicação vigente. 


\section{Resultados}

\section{Capítulo 1 - Como abordar?}

\section{Formas de abordagem comportamental}

A Entrevista Motivacional é um método de aconselhamento em saúde baseado em princípios, espírito e técnicas que, por meio do diálogo, aborda questões de saúde e visa trabalhar a motivação intrínseca das pessoas que estão em processo de mudança, ou seja, ambivalentes. Teve seu início em 1983, sendo um novo método clínico para intervenção em tratamento do alcoolismo. Uma vez que se apresentou como uma abordagem eficiente para mudança de hábitos não saudáveis, em 1990, começou a ser utilizada para o tratamento de doenças crônicas não transmissíveis. A partir do momento que o profissional observa que seu paciente apresenta interesse em fazer alguma modificação, a EM pode ser aplicada explorando essa ambivalência que é parte natural do processo de mudança e não deve ser entendida como patológica nem contraproducente. O profissional deve agir de forma que estimule a reflexão sobre a mudança necessária, através do aumento da motivação intrínseca, e a se desenvolverem em direção a mudança na prática (MARKLAND et al., 2005; MILLER, 1983; MILLER; ROLLNICK, 2001). A EM valida a importância de um ambiente que propicie ao indivíduo ser ativo e criador das decisões a serem tomadas em busca de hábitos melhores, estimulando a autonomia e fortalecendo a autoeficácia do paciente. Dessa forma, o sujeito reconquista a sua confiança por intermédio do diálogo, sendo o profissional capacitado a exercer uma escuta empática, a evocar razões para a mudança e colaborar na construção desse processo levando em consideração os princípios da EM, que são:

- Resistir ao reflexo de "consertar as coisas" do paciente

- Compreender e explorar motivos para a mudança;

- Ser empático ao realizar a escuta;

- Fortalecer o sujeito frente suas razões com otimismo e esperança.
O profissional, ainda, poderá utilizar durante o processo de mudança três estilos de comunicação: acompanhar e escutar; direcionar e orientar. O estilo acompanhar e escutar busca compreender o contexto do indivíduo, sem julgamentos e aconselhamentos, realiza escuta de forma plena e acompanha o momento do paciente; o direcionar promove maior autoridade do profissional por parte das ações, ele conduzirá as combinações por intermédio da relação já construída com o paciente, sendo esperado que o paciente acate ao combinado; e, por último, o estilo orientar auxilia o paciente a encontrar caminhos factíveis a seguir. Esses estilos de comunicação podem variar de acordo com o momento do paciente e, para tal, é necessário que o profissional esteja habilitado a reconhecer esses momentos e que assim possa optar pela melhor abordagem. Portanto, a EM permite que o sujeito perceba suas razões para mudança, despertando a motivação, e com auxílio profissional encontre a melhor maneira para alcançar seu objetivo (ROLLNICK; MILLER; BUTLER, 2009).

Como forma de auxiliar na exploração e resolução da ambivalência e caminhar na direção da mudança de comportamento, o profissional pode utilizar as estratégias iniciais da EM composta por quatro técnicas, conhecidas pelo acrônimo na língua inglesa: OARS (Open-endedquestions, Affirmation, Reflectivelistening, Summaries). A primeira delas é o uso de perguntas abertas, possibilitando ao paciente se expressar livremente e trazer as informações que the parecem pertinentes. A segunda estratégia são afirmações, como reforços positivos, que mantém o paciente em movimento e estimulam a esperança e a autoestima. Uma terceira estratégia é a chamada escuta reflexiva, que é uma forma ativa de escuta, onde se reflete ao paciente pontos estratégicos e interessantes em direção a mudança. Finalmente, há os resumos periódicos, que são úteis para mostrar atenção ao que foi trazido pelo paciente e reforçar os pontos importantes que apareceram em favor da mudança. Na EM, o profissional mais escuta seu paciente do que fala (MILLER, 1983; MILLER; ROLLNICK, 2001). 


\section{APS em Revista}

Vol. 1, n. 3, p. 189/197 | Setembro/Dezembro - 2019

ISSN 2596-3317 - DOI 10.14295/aps.v1i3.43

Faustino-Silva D. D.; Jung, N. M.; Porta, L. L. L.

Outra abordagem comportamental que pode auxiliar no processo de mudança do comportamento alimentar, é o comer intuitivo. 0 comer intuitivo é um conceito criado em 1995 por nutricionistas norte-americanas e baseia-se na perspectiva da importância das sensações fisiológicas, dando vazão aos sinais de fome e de saciedade para o comportamento alimentar (TRIBOLE; ELYSE, 2012) e promovendo hábitos alimentares saudáveis a partir da boa relação dos indivíduos com a comida e o corpo (ALVARENGA et al., 2015). Essa abordagem tem três pilares norteadores: permissão incondicional para comer; comer para atender necessidades fisiológicas e não emocionais e apoiar-se nos sinais internos de fome e saciedade para determinar o quê, quanto e quando comer. $\mathrm{O}$ comer intuitivo propõe 10 princípios:

Tabela 2.Princípios do Comer Intuitivo

\begin{tabular}{|c|c|}
\hline Princípio & Conceito \\
\hline $\begin{array}{l}\text { 1. Rejeitar a } \\
\text { mentalidade de } \\
\text { dieta }\end{array}$ & $\begin{array}{l}\text { Livrar-se de materiais sobre dietas ou calorias que ofereçam a esperança de perda } \\
\text { de peso rápida. Entender que a sensação de fracasso que acontece toda vez que a } \\
\text { dieta é interrompida é causada por quem propôs e não quem tentou segui-la. }\end{array}$ \\
\hline 2.Honrar a fome & $\begin{array}{l}\text { Para honrar a fome é necessário reconhecer os sinais de fome e saciedade e ter } \\
\text { horários padronizados. Deve-se estar aberto para sentir fome sem julgamento e } \\
\text { manter o corpo alimentado, caso contrário favorece o comer em excesso. }\end{array}$ \\
\hline $\begin{array}{l}\text { 3.Fazer as pazes } \\
\text { com a comida }\end{array}$ & $\begin{array}{l}\text { Livrar-se da ideia de que existam alimentos proibidos e permitir-se comer } \\
\text { incondicionalmente, de forma a vivenciar com maior intensidade o momento. }\end{array}$ \\
\hline $\begin{array}{l}\text { 4.Desafiar } 0 \\
\text { policial alimentar }\end{array}$ & $\begin{array}{l}\text { Evitar cobranças internas a respeito de comer alimentos "bons" ou "ruins", evitando } \\
\text { pensamentos disfuncionais dicotômicos ou lineares. }\end{array}$ \\
\hline $\begin{array}{l}\text { 5.Sentir a } \\
\text { saciedade }\end{array}$ & Escutar quando os sinais de saciedade alertarem que se está satisfeito. \\
\hline $\begin{array}{l}\text { 6.Descobrir } \quad \text { o } \\
\text { fator de satisfação }\end{array}$ & Reconhecer alimentos que satisfaçam a fome e o prazer de comer. \\
\hline $\begin{array}{l}\text { 7.Lidar com as } \\
\text { emoções sem } \\
\text { usar comida }\end{array}$ & $\begin{array}{l}\text { Encontrar formas de alívio, estímulo, distração e resolução de problemas que não } \\
\text { sejam comida. Distinguir fome emocional e física. }\end{array}$ \\
\hline $\begin{array}{l}\text { 8.Respeitar seu } \\
\text { corpo }\end{array}$ & Aceitar a genética e aprender a abandonar a ideia de que o corpo é maleável. \\
\hline $\begin{array}{ll}\text { 9. Exercitar-se } & - \\
\text { sentindo } & \text { a } \\
\text { diferença } & \end{array}$ & $\begin{array}{l}\text { Praticar atividade física com o intuito de prazer e saúde, evitando pensamentos } \\
\text { relacionados a gasto energético. }\end{array}$ \\
\hline $\begin{array}{l}\text { 10. Honrar a saúde } \\
\text { - praticar uma } \\
\text { nutrição gentil }\end{array}$ & $\begin{array}{l}\text { Fazer com que as escolhas alimentares sejam realizadas honrando a saúde e o } \\
\text { paladar. }\end{array}$ \\
\hline
\end{tabular}

Fonte: Adaptado de TRIBOLE; ELYSE, 2012 
Comer com atenção plena pode ser descrito como o ato de comer com atenção aos sinais fisiológicos e emocionais, sem culpa e julgamento, trazendo atenção para o presente (FRAMSON et al., 2010). Dessa forma, os indivíduos tornam-se aptos e mais capacitados a realizarem críticas positivas sobre qual forma se alimentam, avaliando o contexto do ambiente e as circunstâncias durante o ato da refeição. Embora a atenção plena seja uma capacidade inata e, possivelmente em virtude de uma imposição vinda por parte da cultura e da sociedade atual, observa-se que as pessoas têm cada vez menos tempo para comer e com diversas distrações (ALVARENGA et al., 2015). Para isso, comer com atenção plena favorece o resgate da atenção sem julgamento ou crítica às sensações despertadas durante o ato de comer (FRAMSON et al., 2010). Apesar de ser uma capacidade inata, a atenção plena pode ser treinada por meio da meditação aguçando a atenção e o foco.

A meditação é uma prática que consiste na habilidade de focar a atenção em algo e não está restrita apenas a monges ou "seres iluminados" como popularmente se acredita. Embora tenha suas raízes no Budismo, a meditação não tem relação com a religião, porém suas características se assemelham com os princípios religiosos. Quando baseada em atenção plena, trata-se de uma técnica simples que pode ser realizada por qualquer pessoa. Para realizá-la é necessário um ambiente tranquilo, em que seja viável sentar-se em uma posição confortável e permitir a libertação dos pensamentos, como descrita na Figura 2 abaixo, a qual orienta uma técnica típica de meditação com duração de um minuto (ALVARENGA et al., 2015; WILLIAMS; PENMAN, 2015).

Sendo a meditação uma técnica capaz de trabalhar a habilidade em atenção plena, sua prática frequente pode se tornar uma atitude positiva para saúde. Uma vez que traz maior reconhecimento dos sentimentos, sem julgamentos ou críticas, a meditação uma atividade que impulsiona sentimentos positivos (WILLIAMS; PENMAN, 2015). Trabalhos sugerem que a prática regular de meditação com atenção plena possa trazer benefícios para ansiedade, depressão e irritabilidade (BAER et al., 2014) tornando as pessoas mais felizes e satisfeitas consigo mesmas (IVANOWSKI; MALHI, 2007; SHAPIRO et al., 2008; SHAPIRO; SCHWARTZ; BONNER, 1998). A partir disso, a meditação pode se tornar também uma ferramenta interessante a ser utilizada em atividades coletivas.

\section{Capítulo 2 - Como fazer?}

Atendimentos em grupo na Atenção Primária são uma forma de realizar educação em saúde para a população, favorecendo o autocuidado, a troca de experiências e o relacionamento entre os indivíduos. O uso de práticas e dinâmicas da abordagem comportamental em grupos de nutrição e/ou alimentação saudável podem ser uma estratégia importante para a mudança de comportamento e para o empoderamento da população.

\section{Linha do Tempo}

Objetivo: refletir sobre a relação entre práticas alimentares adotadas ao longo da vida com o histórico de peso.

Descrição da atividade: o coordenador do grupo deverá selecionar um voluntário para construção de sua linha do tempo alimentar. É importante que se priorize algum participante que já tenha realizado algum tipo de dieta restritiva. A partir das questões norteadoras listadas abaixo, o coordenador irá preencher a linha traçada em um quadro branco ou folha em branco com os relatos do indivíduo:

- Qual o seu peso durante a infância, adolescência e hoje (sinalizar na linha do tempo). Como você se via nessas fases de vida? Acima do peso, baixo peso, normal, etc.

- Você identifica algum momento de sua vida em que tenha ocorrido alguma mudança importante de peso? Qual episódio você associa a essa mudança? Por exemplo, ao casar, ao engravidar, mudança de residência. Você realizou algum procedimento cirúrgico que resultou em perda/ganho de peso? 
- Coloque na linha do tempo os momentos que você praticou dietas restritivas, hábitos alimentares adversos ou alguma prática relacionada à perda de peso. Como era seu peso antes e depois desses eventos? Você recorda como se sentia? (Exemplo: estressada, animada, nervosa, motivada)

- Se você realizou atividade física regular, inclua na linha do tempo o período, a idade e o peso.

- Você já realizou reeducação alimentar ou teve acompanhamento de nutricionista? Insira na linha do tempo a época e indique seu peso de início e final.

- Além daquilo que foi questionado, você recorda alguma outra situação que ache importante incluir na sua linha do tempo?

Ao final, o coordenador deverá fazer um resumo da linha do tempo e, em conjunto ao grupo analisar as alterações de peso de acordo com práticas e o efeito das dietas restritivas.

Materiais: quadro branco e pincel atômico ou caneta hidrocor e folha branca.

Resultados esperados: elucidação do efeito de dietas restritivas no efeito sanfona, propiciando a reflexão acerca de estratégias mais eficazes para perda de peso que são

\section{Selecionando Alimentos}

Objetivo: promover autoconhecimento a respeito de suas escolhas e preferências alimentares

Descrição da atividade: cada participante receberá uma folha dividida em quatro partes: (1) coisas que como e gosto (2) coisas que como e não gosto, (3) coisas que não como e gosto e (4) coisas que não como e não sei se gosto. Serão instruídos a pensar sobre alimentos que se encaixem em cada item além de citar vantagens e desvantagens para cada escolha de cada um. Trabalhar com a ideia da balança decisional, ou seja, pesar ganhos e prejuízos nas decisões alimentares.
Materiais: folha em branco e caneta esferográfica.

Resultados esperados: refletir sobre excessos, restrições e padrões alimentares que interferem na relação com a comida. Também, observar e explorar se ocorre a monotonia alimentar, sendo propiciadas oportunidades para conhecer e aprender a degustar novos sabores.

\section{Que fome sou eu?}

Objetivo: trabalhar os conceitos de fome e discutir a respeito da sua ocorrência no dia-a-dia

Descrição da atividade: os participantes devem se organizar em um círculo. Uma caixa com questões percorrerá o círculo e cada integrante deverá retirar uma pergunta. Dessa forma, cada participante terá em mãos uma questão, que será lida em voz alta. Uma caixa com respostas percorrerá o círculo e cada integrante deverá retirar uma resposta. Quem tiver retirado uma resposta e julgar correta, deverá ler em voz alta. O coordenador deverá estimular a discussão, trazendo experiências, relatos, de forma a refletir sobre a mistura dos tipos de fome que são sentidas habitualmente. Ainda, questionar se algum participante se identifica mais com algum tipo de fome e se existem situações ou momentos que possam acontecer mais.

A atividade inicia com qualquer questão e não possui ordem correta, apenas respostas correspondentes às questões.

Assertiva 1: Estou relacionada com sentimentos. Quando me sinto muito triste ou muito feliz, eu como para viver aquele momento. Geralmente nesse momento quero comer um alimento bem gostoso e não sei definir qual é. Que tipo de fome eu sou?

Assertiva 2: Sou relacionada com o prazer e o sabor, por exemplo: depois do almoço, sinto fome de um alimento específico. Que tipo de fome eu sou?

Assertiva 3: Sou aquela fome que vive no automático e que acompanhada de outras pessoas não percebo o quê e nem quanto estou comendo. $O$ 
Vol. 1, n. 3, p. 189/197 | Setembro/Dezembro - 2019

ISSN 2596-3317 - DOI 10.14295/aps.v1i3.43

Faustino-Silva D. D.; Jung, N. M.; Porta, L. L. L.

meu normal é comer mais que quando estou sozinha. Que tipo de fome eu sou?

Assertiva 4: Quando a barriga ronca, preciso comer qualquer alimento. Algumas pessoas sentem dor de cabeça quando estou presente. Que tipo de fome eu sou?

Resposta da assertiva 1: Eu sou a fome emocional.

- Hoje estou muito triste, acho que mereço comer algo bem gostoso.

Resposta da assertiva 2: Eu sou a vontade de comer ou fome específica.

- Sinto uma vontade de comer mousse de maracujá.

Resposta da assertiva 3: Eu sou a fome social.

- Nem sei dizer quantos docinhos comi nessa festa de aniversário.

- Sinto-me empanturrada quando vou à pizzaria. Sempre como além do que gostaria.

Resposta da assertiva 4: Eu sou a fome fisiológica.

- Eu comeria qualquer coisa agora só para matar essa fome.

Materiais: caixa de papelão de tamanho médio, dicas e respostas impressas em papel e recortadas.

Resultados esperados: aprendizado sobre os conceitos de fome e o reconhecimento de situações gatilho para os tipos de fome.

\section{CONCLUSÃo}

Uma vez que o excesso de peso e a obesidade são problemas de saúde pública, tornase importante a busca por estratégias de tratamento efetivas. Sendo a abordagem comportamental aplicada à nutrição uma possibilidade eficaz de cuidado, sugere-se sua aplicabilidade na Atenção Básica. Dessa maneira, o vigente trabalho teve como objetivo a construção de um material científico voltado para os profissionais, que sirva de apoio em atividades coletivas na Atenção Básica. Com o intuito de prover conhecimento teórico e prático, os profissionais poderão trabalhar o cuidado em saúde a partir de uma nova abordagem.

\section{REFERÊNCIAS BIBLIOGRÁFICAS}

ABESO. Diretrizes brasileiras de obesidade 2016. 4. ed. São Paulo: [s.n.].

ALVARENGA, M. et al. Nutrição Comportamental. $1^{\text {a }}$ Edição ed. São Paulo: Manole, 2015.

BAER, R. et al. Using Self-Report Assessment Methods to Explore Facets of Mindfulness. n. April 2006, 2014.

BRASIL. MINISTÉRIO DA SAÚDE. Secretaria de Atenção à Saúde. Departamento de Atenção Básica. Obesidade / Ministério da Saúde, Secretaria de Atenção à Saúde, Departamento de Atenção Básica. - Brasília : Ministério da Saúde, 2006.

BRASIL. MINISTÉRIO DO DESENVOLVIMENTO SOCIAL E COMBATE À FOME. Marco de referência de educação alimentar e nutricional para as políticas públicas. Brasília: MDS; Secretaria Nacional de Segurança Alimentar e Nutricional, 2012.

BUTLAND, B. et al. Foresight Tackling Obesities: Future Choices - Project report. Government Office for Science, p. 1-161, 2007.

FRAMSON, C. et al. Development and Validation of the Mindful Eating Questionnaire. v. 109, n. 8, p. 1439-1444, 2010.

IVANOWSKI, B.; MALHI, G. S. The psychological and neurophysiological concomitants of mindfulness forms of meditation. Acta Neuropsychiatrica, p. 19, 2007.

MILLER, W. R. Motivational interviewing with problem drinkers. Behavioural Psychotherapy. London, v. 11, n. 2, p. 147-172, 1983

MILLER, W. R.; ROLLNICK, S. Entrevista motivacional: preparando as pessoas para a mudança de 
APS em Revista

Vol. 1, n. 3, p. 189/197 | Setembro/Dezembro - 2019

ISSN 2596-3317 - DOI 10.14295/aps.v1i3.43

Faustino-Silva D. D.; Jung, N. M.; Porta, L. L. L.

comportamentos adictivos. 1. ed. Porto Alegre:

Artmed, 2001

ROLLNICK, S.; MILLER, W.; BUTLER, C. Entrevista Motivacional no Cuidado da Saúde. 2007.

SHAPIRO, S. L. et al. Cultivating Mindfulness : Effects on Well-Being. v. 64, n. 7, p. 840-862, 2008.

SHAPIRO, S. L.; SCHWARTZ, G. E.; BONNER, G. Effects of Mindfulness-Based Stress Reduction on Medical and Premedical Students. v. 21, n. 6, p. 581-600, 1998.

TRIBOLE, E.; ELYSE, R. Intuitive Eatings - A revolutionary program that works. New York, 2003.

WILLIAMS, M.; PENMAN, D. Atenção Plena. 2011.

WORLD HEALTH ORGANIZATION. OBESITY: PREVENTING AND MANAGING THE GLOBAL EPIDEMIC Report of a WHO Consultation. 2000. 


\section{ABSTRACT}

Background: The rising prevalence of overweight and obesity is a worldwide reality. The search for new treatment strategies becomes important since conventional approaches may not be effective. The behavioral approach in nutrition may be an alternative as a new form of care since it also allows a greater understanding of the behavior related to the act of overeating. Thus, the objective of the work was the elaboration of a scientific material that promotes knowledge about the behavioral approach applied in nutrition aimed at people with obesity.

Methods: Bibliographic review, content summary, adaptation and creation of didactic material.

Results: Based on the theoretical review, a booklet was developed, with theoretical and practical elements, which aims to offer theoretical and practical material on behavioral approach and the application of its techniques to obese patients in the context of collective health actions in PHC.

Conclusions: The development of scientific material promotes applied knowledge in nutrition for people with obesity

Keywords: Primary Health Care; Eating behavior; Obesity; Food and Nutrition Education; Cognitive behavioral therapy. 